\title{
FURTHER REDUCTIONS OF POINCARÉ-DULAC NORMAL FORMS IN $\mathbf{C}^{n+1}$
}

\author{
ADRIAN JENKINS
}

(Communicated by Mei-Chi Shaw)

\begin{abstract}
In this paper, we will consider (germs of) holomorphic mappings of the form $\left(f(z), \lambda_{1} w_{1}\left(1+g_{1}(z)\right), \ldots, \lambda_{n} w_{n}\left(1+g_{n}(z)\right)\right)$, defined in a neighborhood of the origin in $\mathbf{C}^{n+1}$. Most of our interest is in those mappings where $f(z)=z+a_{m} z^{m}+\cdots$ is a germ tangent to the identity and $g_{i}(0)=0$ for $i=$ $1, \ldots, n$, and $\lambda_{i} \in \mathbf{C}$ possess no resonances, for these are the so-called PoincaréDulac normal forms of the mappings $\left(z+O(2), \lambda_{1} w+O(2), \ldots, \lambda_{n} w+O(2)\right)$. We construct formal normal forms for these mappings and discuss a condition which tests for the convergence or divergence of the conjugating maps, giving specific examples.
\end{abstract}

\section{INTRODUCTION}

The purpose of this paper is two-fold. On the one hand, we complete the formal classification of semihyperbolic mappings of $\mathbf{C}^{n+1}$ which fix the origin and whose eigenvalues possess no additional resonance. This classification was begun by Poincaré and Dulac in the early part of the 20th century, and the problem of a complete formal classification of such mappings was raised in a survey paper of Abate [1]. On the other hand, we show that the formal and local holomorphic classification of such mappings differ wildly, a fact that is anticipated from the onevariable results of Voronin, Malgrange and Il'yashenko (see [5] or [8]). In fact, we will develop specific criteria to determine the convergence or divergence of formal conjugating maps between holomorphic mappings. A goal of this paper is to construct links between the well-understood local theory of one complex variable and the comparatively unknown theory in several variables.

Let us first fix notation and give preliminary definitions. Throughout the paper, we will define the $j^{\text {th }}$ iterate of a mapping $F$ as $F^{\circ j}$. We consider (germs of) holomorphic mappings $F$ of $(n+1)$ variables fixing a point $p \in \mathbf{C}^{n+1}$. After a change of variable, we will always assume that $p=0$. We say that $F$ is semihyperbolic if $F$ can be written, near zero, as

$$
F\left(z, w_{1}, \ldots, w_{n}\right)=\left(z+O(2), \lambda_{1} w_{1}+O(2), \ldots, \lambda_{n} w_{n}+O(2)\right),
$$

where $0<\left|\lambda_{j}\right| \neq 1$ for all $j=1, \ldots, n$. We will say that the eigenvalues $\lambda_{1}, \ldots, \lambda_{n}$ possess additional resonance if, for any $k$, we can write $\lambda_{k}=\prod_{j=1}^{n} \lambda_{j}^{m_{j}}$, where $m_{j} \in$ $\mathbf{N} \cup\{0\}$ and $\sum_{j=1}^{n} m_{j} \geq 2$ (the nonstandard adjective "additional" is in regards to

Received by the editors August 28, 2006, and, in revised form, December 11, 2006. 2000 Mathematics Subject Classification. Primary 32A05, 32H50; Secondary 30D05.

Key words and phrases. Holomorphic mappings, conjugacy, equivalence.

(C) 2008 American Mathematical Society 1671 
the fact that, if we include the eigenvalue 1 , then of course such resonances exist). Finally, we say that two mappings $F$ and $\widetilde{F}$ of the form (1.1) are (formally, locally holomorphically) conjugate if there is a (formal, holomorphic in a neighborhood of 0) mapping $H$ so that $H^{-1} \circ F \circ H=\widetilde{F}$. Unless otherwise noted, all of our conjugating maps will be taken to be tangent to the identity.

In the absence of any additional resonance, the well-known formal theory of Poincaré and Dulac (see, e.g., [3]) shows that any mapping $F$ of the form (1.1) may be formally conjugated to the mapping

$$
F_{0}\left(z, w_{1}, \ldots, w_{n}\right)=\left(f(z), \lambda_{1} w_{1}\left(1+g_{1}(z)\right), \ldots, \lambda_{n} w_{n}\left(1+g_{n}(z)\right)\right),
$$

where $f(z)=z+a_{m} z^{m}+\cdots$, and $g_{j}(0)=0$. By a linear change of coordinates, we will always assume that $a_{m}=1$, and so we will consider mappings

$$
f(z)=z+z^{m}+\cdots, \quad g_{j}(z)=b_{j, k} z^{k}+\cdots,
$$

where $b_{j, k} \neq 0$ is the first nonzero coefficient of $g_{j}$ for all $j=1, \ldots, n$.

The formal and holomorphic theory of mappings tangent to the identity in $(\mathbf{C}, 0)$ is well known; we will make use of the following two results. The first result is very well known; a proof of this fact may be found in [6].

Result 1. There exists $\mu \in \mathbf{C}$ so that any (possibly formal) map of the form $f(z)=z+z^{m}+\cdots$ may be formally conjugated (via a formal map of the form $h(z)=z+\cdots)$ to $f_{0}(z)=z+z^{m}+\mu z^{2 m-1}$.

The next result was proven by Camacho [4] and Shcherbakov [7], and relates to topological conjugacy:

Result 2. Suppose that $f(z)=z+z^{m}+\cdots$. Then, there is a neighborhood $U$ of 0 and a homeomorphism $h(z)=z+\hat{h}(z)$ so that $h^{-1} \circ f \circ h=g_{V_{m}}^{1}$, where $g_{V_{m}}^{1}$ is the time-one map of the vector field $V_{m}(z)=z^{m} \frac{\partial}{\partial z}$. Moreover, given any $\varepsilon>0$, we can choose $U$ and $\hat{h}$ so that $\hat{h}(z) \leq|z|^{m-\varepsilon}$.

An important consequence of Result 2 is the following: for $k=1, \ldots, 2(m-1)$, define the sectors

$$
S_{k}=\left\{z \in \mathbf{C}:|z|<r,\left|\arg z-\frac{\pi k}{m-1}\right|<\frac{\pi}{2(m-1)}+\delta\right\},
$$

where $0<r \ll 1$ and $0<\delta<\frac{\pi}{2(m-1)}$. By choosing $r$ small enough and possibly shrinking $\delta$, the topological equivalence of Result 2 shows that the sector $S_{k}$ is attracting for the function $f$ whenever $k$ is odd, and repelling whenever $k$ is even.

As original results, we prove the following two theorems. The first completes the formal classification of semihyperbolic mappings of $\mathbf{C}^{n+1}$ :

Theorem 1.1. Given a (possibly formal) mapping $F$ of the form (1.2), where $f$ and $g_{i}$ are of the form (1.3), there exists a formal mapping $H$ given by

$$
H\left(z, w_{1}, \ldots, w_{n}\right)=\left(h(z), w_{1} k_{1}(z), \ldots, w_{n} k_{n}(z)\right),
$$

where $h(0)=0, h^{\prime}(0)=1$ and $k_{i}(0)=1$ for each $i=1, \ldots, n$, which conjugates $F$ with the mapping

$F_{0, m, \mu}\left(z, w_{1}, \ldots, w_{n}\right)=\left(z+z^{m}+\mu z^{2 m-1}, \lambda_{1} w_{1}\left(1+g_{1}^{*}(z)\right), \ldots, \lambda_{n} w_{n}\left(1+g_{n}^{*}(z)\right)\right)$,

where $m \in \mathbf{N}-\{1\}$ and $\mu \in \mathbf{C}$ are the (one-variable) formal invariants of $f$, and $g_{i}^{*}$ is a (holomorphic) polynomial of degree at most $m-1$ for each $i=1, \ldots, n$. 
Moreover, if no additional resonances are present among the eigenvalues of $F$, the polynomials $g_{i}^{*}$ provide formal invariants for the mapping $F$.

In other words, Theorem 1.1, together with the classical results of Poincaré and Dulac, provides a complete formal normal form for mappings of the form (1.1). Note that the omission of resonance is only necessary to ensure that the mapping $F_{0, m, \mu}$ is in fact a normal form; the existence of a conjugating map $H$ does not depend on this hypothesis.

We then prove a criterion to determine the convergence or divergence of such normalizing maps $H$, and provide specific examples of divergence.

Theorem 1.2. Suppose that

$$
F\left(z, w_{1}, \ldots, w_{n}\right)=\left(f(z), \lambda_{1} w_{1}\left(1+g_{1}(z)\right), \ldots, \lambda_{n} w_{n}\left(1+g_{n}(z)\right)\right)
$$

and

$$
\widetilde{F}\left(z, w_{1}, \ldots, w_{n}\right)=\left(\tilde{f}(z), \lambda_{1} w_{1}\left(1+\tilde{g}_{1}(z)\right), \ldots, \lambda_{n} w_{n}\left(1+\tilde{g}_{n}(z)\right)\right)
$$

are two holomorphic mappings which are holomorphically conjugated by a mapping $H$ of the form (1.5). Then, the following two equations must be satisfied:

i) $h^{-1} \circ f \circ h=\tilde{f}$,

ii) $0=\sum_{j=-\infty}^{\infty} \log \left(\frac{1+\left(g_{i} \circ h \circ f^{\circ j}\right)}{1+\left(\tilde{g}_{i} \circ f^{\circ j}\right)}\right)$.

Equation (i) must be true for each $i=1, \ldots, n$. On the other hand, if $f$ and $\tilde{f}$ can be holomorphically conjugated by a mapping $h$ defined in a full neighborhood of $0 \in \mathbf{C}$ so that equation (ii) is satisfied, then $F$ and $\widetilde{F}$ are holomorphically equivalent.

Equation (i) in Theorem 1.2 shows that any holomorphic normalization is dependent on the well-known one-variable holomorphic normalization (which differs significantly from the formal normalization; see [5] or [8]), so the interest here is on equation (ii).

Theorem 1.1 and part of Theorem 1.2 were part of the author's thesis, written under the direction of Xianghong Gong at the University of Wisconsin-Madison. The author is thankful to Professor Gong for interest in, and discussion on, the problems presented here.

\section{Formal THEORY}

We prove here Theorem 1.1. The following lemma shows that the mappings $H$ given in Theorem 1.1 are of the correct form, provided that there are no additional resonances present between the eigenvalues $\lambda_{1}, \ldots, \lambda_{n}$.

Lemma 2.1. Suppose that

$$
F\left(z, w_{1}, \ldots, w_{n}\right)=\left(f(z), \lambda_{1} w_{1}\left(1+g_{1}(z)\right), \ldots, \lambda_{n} w_{n}\left(1+g_{n}(z)\right)\right)
$$

and

$$
\widetilde{F}\left(z, w_{1}, \ldots, w_{n}\right)=\left(\tilde{f}(z), \lambda_{1} w_{1}\left(1+\tilde{g}_{1}(z)\right), \ldots, \lambda_{n} w_{n}\left(1+\tilde{g}_{n}(z)\right)\right)
$$

are formal mappings, where $f$ and $\tilde{f}$ are both tangent to the identity, $g_{i}(z)=$ $\sum_{k=1}^{\infty} b_{i k} z^{k}$, and $\tilde{g}_{i}(z)=\sum_{k=1} b_{i k} z^{k}$. Then, given any formal mapping $H$ conjugating $F$ with $\widetilde{F}$, we have

$$
H\left(z, w_{1}, \ldots, w_{n}\right)=\left(h_{1}(z), w_{1} k_{1}(z), \ldots, w_{n} k_{n}(z)\right),
$$


where $k_{i}(0) \neq 0$ for all $i=1, \ldots, n$, provided that no resonances exist between the eigenvalues $\lambda_{1}, \ldots, \lambda_{n}$.

Proof. For notational convenience, we assume that $n=1$; the general case can be proven in a similar (though lengthier) manner. We note that if $F \circ H=H \circ \widetilde{F}$, then we have the following functional equations:

i) $f\left(h_{1}(z, w)\right)=h_{1}(\tilde{f}(z), \lambda w(1+\tilde{g}(z)))$,

ii) $\lambda h_{2}(z, w)\left(1+g\left(h_{1}(z, w)\right)\right)=h_{2}(\tilde{f}(z), \lambda w(1+\tilde{g}(z)))$.

Let us write $h_{1}(z, w)=\sum_{m+n=1}^{\infty} c_{m n} z^{m} w^{n}$ and $h_{2}(z, w)=\sum_{s+t=1}^{\infty} d_{s t} z^{s} w^{t}$. We work first with equation i), which we now write as

$$
\sum_{m+n=1}^{\infty} c_{m n}(\tilde{f}(z))^{m}(\lambda w)^{n}(1+g(z))^{n}=\sum_{m+n=1}^{\infty} c_{m n} z^{m} w^{n}+\cdots
$$

We first show that $c_{0 n}=0$ for all $n$, via induction on $n$. For $n=1$, we have $c_{01} w=c_{01}(\lambda w)$. Since $\lambda$ is not a root of unity, this implies that $c_{01}=0$. In general, we can write

$$
c_{0 n} w^{n}+\left(\sum_{j=1}^{n-1} \alpha_{j} c_{0 j}\right) w^{n}=c_{0 n}(\lambda w)^{n},
$$

where $\alpha_{j} \in \mathbf{C}$. By the induction hypothesis, (2.5) reduces to $c_{0 n} w^{n}=c_{0 n}(\lambda w)^{n}$, which then implies that $c_{0 n}=0$ for all $n=1,2, \ldots$. From here, it is easy to show that $c_{m n}=0$ for all $m=0,1, \ldots$ and $n=1,2, \ldots$, by induction on $m$ for every $n$. Hence, the only terms which can possibly survive are those with coefficients of the form $c_{m 0}$, that is, $h_{1}(z, w)=h(z)$.

With this fact in hand, we write equation ii) in the form

$$
\lambda\left(\sum_{s+t=1}^{\infty} d_{s t} z^{s} w^{t}\right)(1+g(h(z)))=\sum_{s+t=1}^{\infty} d_{s t}(\tilde{f}(z))^{s}(\lambda w)^{t}(1+\tilde{g}(z))^{t} .
$$

Here, we show that $d_{s t}=0$ for all $t \neq 1$. We apply induction on $s$, beginning at $t=0$. For $s=1$, we have $d_{10} z=\lambda d_{10} z$, implying that $d_{10}=0$. In general, we have

$$
d_{s 0} z^{s}+\left(\sum_{j=1}^{s-1} \beta_{j} d_{j 0}\right) z^{s}=\lambda d_{s 0} z^{s}+\left(\sum_{j=1}^{s-1} \tilde{\beta}_{j} d_{j 0}\right) z^{s}
$$

where again $\beta_{j}, \tilde{\beta}_{j} \in \mathbf{C}$. By the induction hypothesis, we see that $d_{s 0}=0$ for all $s$. Applying the same idea, we can show that $d_{s t}=0$ provided that $t \neq 1$. Therefore, we can write $h_{2}(z, w)=w k(z)$. This proves the lemma.

Of course, any holomorphic mapping $H$ conjugating $F$ and $\widetilde{F}$ of the forms (2.1) and (2.2), resp., must also take the form (2.3) as well.

We now complete the proof of Theorem 1.1 by proving the classification given there. Given a mapping $F$ of the form (1.2), we begin by normalizing $f$. By Result 1 , we can take $f$, via a formal transformation $h$, to the normal form $\tilde{f}(z)=z+z^{m}+$ $\nu z^{2 m-1}$. This normalizing transformation necessarily has the form $h(z)=z+\cdots$, and thus, if we define the formal mapping

$$
H_{1}\left(z, w_{1}, \ldots, w_{n}\right)=\left(h(z), w_{1}, \ldots, w_{n}\right),
$$


then conjugation of $F$ by $H$ yields the mapping

$$
\begin{aligned}
\widetilde{F}\left(z, w_{1}, \ldots, w_{n}\right) & =\left(H^{-1} \circ F \circ H\right)\left(z, w_{1}, \ldots, w_{n}\right) \\
& =\left(h^{-1} \circ f \circ h(z), \lambda_{1} w_{1}\left(1+g_{1} \circ h(z)\right), \ldots, \lambda_{n} w_{n}\left(1+g_{n} \circ h(z)\right)\right) \\
& =\left(z+z^{m}+\nu z^{2 m-1}, \lambda_{1} w_{1}\left(1+\tilde{g}_{1}(z)\right), \ldots, \lambda_{n} w_{n}\left(1+\tilde{g}_{n}(z)\right)\right) .
\end{aligned}
$$

We now eliminate all terms of order greater than $m-1$ in each formal map $\tilde{g}_{i}$, $i=1, \ldots, n$. We will consider a formal map of the form

$$
H_{2}\left(z, w_{1}, \ldots, w_{n}\right)=\left(z, w_{1} k_{1}(z), \ldots, w_{n} k_{n}(z)\right),
$$

where $k_{i}(0)=1$ for $i=1, \ldots, n$. It is clear that $H_{2}$ is formally invertible, with inverse

$$
H_{2}^{-1}\left(z, w_{1}, \ldots, w_{n}\right)=\left(z, \frac{w_{1}}{k_{1}(z)}, \ldots, \frac{w_{n}}{k_{n}(z)}\right)
$$

We again assume $n=1$ for notational convenience. Conjugating $\widetilde{F}$ by $H_{2}$, we obtain

$$
\begin{aligned}
\left(H_{2}^{-1} \circ \widetilde{F} \circ H_{2}\right)(z, w) & =H_{2}^{-1}\left(z+z^{m}+\nu z^{2 m-1}, \lambda w k(z) \tilde{g}(z)\right) \\
& =\left(z+z^{m}+\nu z^{2 m-1}, \lambda w \frac{k(z)}{k\left(z+z^{m}+\nu z^{2 m-1}\right)} \tilde{g}(z)\right) .
\end{aligned}
$$

We consider polynomials of the form $k(z)=1+c_{j} z^{j}$, where each $c_{j} \in \mathbf{C}$ is to be determined. If $\hat{g}(z)$ is another formal power series in $z$, then (2.12) yields the equation

$$
k(z) \tilde{g}(z)=k\left(z+z^{m}+\nu z^{2 m-1}\right) \hat{g}(z) .
$$

Writing $\tilde{g}(z)=1+\sum_{n=1}^{\infty} a_{n} z^{n}$ and $\hat{g}(z)=1+\sum_{n=1}^{\infty} b_{n} z^{n}$, Equation (2.13) in turn yields the following equations on the coefficients:

i) $a_{n}=b_{n}$ for $n<m+j-1$,

ii) $a_{m+j-1}+c_{j} a_{m-1}=b_{m+j-1}+m c_{j}+b_{m-1} c_{j}$.

Equation ii) can then be solved to eliminate all terms of $\tilde{g}$ with degree greater than $m-1$. Finally, we note that any formal mapping $h$ centralizing $f_{0}(z)=$ $z+z^{m}+\mu z^{2 m-1}$ must take the form $h(z)=z+a_{m} z^{m}+\cdots$, and so Lemma 2.1, together with (2.12), shows that it is generally impossible to eliminate any term of $\tilde{g}$ with degree less than $m$, completing the proof of Theorem 1.1. This completes the formal theory for mappings of the type (1.1).

Remark 2.2. Given two formal mappings $F$ and $\widetilde{F}$ of the form (1.2) which are identical in the first component and possess no resonances between the eigenvalues $\lambda_{1}, \cdots, \lambda_{n}$, then if $F$ is formally equivalent to $\widetilde{F}$, we can always find a formal mapping $H$ of the form

$$
H\left(z, w_{1}, \ldots, w_{n}\right)=\left(z, w_{1} k_{1}(z), \ldots, w_{n} k_{n}(z)\right),
$$

where $k_{i}(0)=1$ so that $H^{-1} \circ F \circ H=\widetilde{F}$.

That is, $H$ may be chosen to be the identity in the first component. Since $F$ and $\widetilde{F}$ must have the same formal normal form (call this $F_{0}$ ), and agree in the first 
component, we can choose conjugating mappings $H$ and $\widetilde{H}$ agreeing in the first component as well:

$$
\begin{aligned}
& H\left(z, w_{1}, \ldots, w_{n}\right)=\left(h(z), w_{1} k_{1}(z), \ldots, w_{n} k_{n}(z)\right), \\
& \widetilde{H}\left(z, w_{1}, \ldots, w_{n}\right)=\left(h(z), w_{1} \tilde{k}_{1}(z), \ldots, w_{n} \tilde{k}_{n}(z)\right) .
\end{aligned}
$$

Then, $\widetilde{H}^{-1} \circ H$ conjugates $F$ with $\widetilde{F}$ formally, and has the desired form. The same cannot be said in the case of a convergent conjugating map, as we shall see shortly.

\section{Convergence And Divergence CRIteria}

In this section, we prove Theorem 1.2 and give some examples of divergent conjugating maps. We begin by proving the necessity of the conditions present there (this proof is quite simple). Suppose that

$$
F\left(z, w_{1}, \ldots, w_{n}\right)=\left(f(z), \lambda_{1} w_{1}\left(1+g_{1}(z)\right), \ldots, \lambda_{n} w_{n}\left(1+g_{n}(z)\right)\right)
$$

and

$$
\widetilde{F}\left(z, w_{1}, \ldots, w_{n}\right)=\left(\tilde{f}(z), \lambda_{1} w_{1}\left(1+\tilde{g}_{1}(z)\right), \ldots, \lambda_{n} w_{n}\left(1+\tilde{g}_{n}(z)\right)\right)
$$

are equivalent via a holomorphic mapping $H$ given by

$$
H\left(z, w_{1}, \ldots, w_{n}\right)=\left(h(z), w_{1} k_{1}(z), \ldots, w_{n} k_{n}(z)\right)
$$

where $k_{i}(0) \neq 0$ (and without loss of generality, we assume that $k_{i}(0)=1$ ). We write $G_{i}(z)=\frac{1+\left(g_{i} \circ h\right)(z)}{1+\tilde{g}_{i}(z)}$. The equation $H^{-1} \circ F \circ H=\widetilde{F}$ yields the two functional equations $h^{-1} \circ f \circ h=\tilde{f}$ and

$$
\frac{\left(k_{i} \circ \tilde{f}\right)(z)}{k_{i}(z)}=G_{i}(z)
$$

for $i=1, \ldots, n$. This immediately shows that equation (i) must be satisfied, so we focus on (3.4). If $k_{i}$ is a holomorphic function, then in the region of attraction $\Omega$ of $\tilde{f}$, we can iterate (3.4) by $\tilde{f}$ as often as we like. Thus, after taking logs and summing over all $n \in \mathbf{N}$, we obtain

$$
-\log k_{i}(z)=\sum_{j=0}^{\infty} \log \left(G_{i} \circ \tilde{f}^{\circ j}\right)(z) .
$$

In like manner, we can work in the region of repulsion $\widetilde{\Omega}$ of $\tilde{f}$ (i.e., the region of attraction of $\tilde{f}^{-1}$ ) and thus obtain the equation

$$
\log k_{i}(z)=\sum_{j=1}^{\infty} \log \left(G_{i} \circ f^{\circ(-j)}\right)(z) .
$$

Finally, we combine Equations (3.5) and (3.6) to complete the proof.

Note that the equation $h^{-1} \circ f \circ h=\tilde{f}$ immediately implies that the formal classification and the holomorphic classification cannot coincide (since they do not coincide in the one variable case; again, see [5] or [8]). However, even in the case in which the first components $f$ and $\tilde{f}$ of $F$ and $\widetilde{F}$ can be holomorphically conjugated (e.g. when $f=\tilde{f}$ ), we can use Theorem 1.2 to construct examples of divergence. 
Example 3.1. Consider the two mappings

$$
F(z, w)=\left(\frac{z}{1-z}, \lambda w \exp \left(z^{2}\right)\right) \quad \widetilde{F}(z, w)=\left(\frac{z}{1-z}, \lambda w\right)
$$

where $\lambda$ is not a root of unity. Then, $F$ and $\tilde{F}$ are formally, but not holomorphically, equivalent.

By Theorem 1.1, it is clear that these mappings are formally equivalent via a mapping of the form $H(z, w)=(h(z), w k(z))$. However, since the mapping $h$ centralizes $f(z)=\frac{z}{1-z}$ (the time-one map of the vector field $V(z)=z^{2} \frac{\partial}{\partial z}$ ), we must have that $h(z)=\frac{z}{1-\alpha z}$ for some $\alpha \in \mathbf{C}$ ([5], [8]). The sum in Theorem 1.2 can be written

$$
\sum_{j=-\infty}^{\infty} \log \exp \left(\frac{z}{1-(\alpha+j) z}\right)^{2}=\sum_{j=-\infty}^{\infty}\left(\frac{z}{1-(\alpha+j) z}\right)^{2} .
$$

It is clear that, if we consider the sectors $S_{1}$ and $S_{2}$ given in (1.4), then this quantity is nonzero in the region $S_{1} \cap S_{2}$ (the right-hand side of Equation (3.8) defines a meromorphic function on $\mathbf{C}-\{0\}$, with poles at $\left.z=\frac{1}{\alpha+j}\right)$. Hence, any such conjugating map $H$ must diverge.

As Remark 2.2 indicates, given two holomorphic mappings $F$ and $\widetilde{F}$ of the form (1.2) which are formally equivalent, and which agree in the first component, then one may construct a formal conjugating map $H$ which is the identity in the first component. However, if $F$ and $\widetilde{F}$ are actually holomorphically equivalent, this is no longer true, as the next example demonstrates:

Example 3.2. Consider the two holomorphic mappings given by

$$
F(z, w)=\left(\frac{z}{1-z}, \lambda w \exp (z)\right) \quad \widetilde{F}(z, w)=\left(\frac{z}{1-z}, \lambda w \exp \left(\frac{z}{1-\alpha z}\right)\right),
$$

where $0<|\lambda| \neq 1$ and $0 \neq \alpha \in \mathbf{C}-\mathbf{R}$. Then, $F$ and $\widetilde{F}$ cannot be conjugated holomorphically by a mapping $H$ of the form $H(z, w)=(z, w k(z))$.

That these two mappings are holomorphically equivalent is easily seen (via the transformation $\left.H(z, w)=\left(\frac{z}{1+\alpha z}, w\right)\right)$. However, if $H(z, w)=(z, w k(z))$, the sum given in Theorem 1.2 is written as

$$
\alpha z^{2} \sum_{j=-\infty}^{\infty} \frac{1}{(1-j z)(1-(\alpha+j) z)} .
$$

It is easy to check that this fails to be zero in $S_{1} \cap S_{2}$.

Thus, Theorem 1.2 may be used both to determine the divergence of a conjugating map $H$, or to determine its possible form, as Examples 3.1 and 3.2 illustrate. We remark that since holomorphic conjugating maps in one complex variable are not unique (obviously; if $h$ conjugates $f$ and $\tilde{f}$, and if $g$ is any holomorphic map centralizing $f$, then $g \circ h$ will also conjugate $f$ and $\tilde{f}$ ), and since determining conjugacy is often a difficult task (as noted by Ahern and Rosay, [2]), a full holomorphic classification may be quite delicate.

We now prove the second part of Theorem 1.2. Letting $n=1$ (since the normalization of the $i^{t h}$ component will not depend on the $j^{\text {th }}$ component, if $i, j \geq 2$ ), 
we suppose that $F$ and $\widetilde{F}$ are of the form (1.2), and are formally equivalent. By Result 1 , we may assume that $F$ and $\widetilde{F}$ have the form

$$
\begin{aligned}
& F(z, w)=\left(z+z^{m}+\mu z^{2 m-1}+a_{2 m} z^{2 m}+\cdots, \lambda w(1+g(z))\right), \\
& \widetilde{F}(z, w)=\left(z+z^{m}+\mu z^{2 m-1}+\tilde{a}_{2 m} z^{2 m}+\cdots, \lambda w(1+\tilde{g}(z))\right) .
\end{aligned}
$$

Since $F$ and $\widetilde{F}$ are formally equivalent, we see that the $(m-1)$-jet of $g$ agrees with that of $\tilde{g}$; let us call this jet $g^{*}$.

We now choose a holomorphic mapping tangent to the identity so that $h^{-1} \circ f \circ$ $h=\tilde{f}$ in a full neighborhood of the origin, and so that

$$
1=\prod_{j=-\infty}^{\infty} \frac{1+\left(g \circ h \circ \tilde{f}^{\circ j}\right)(z)}{1+\left(\tilde{g} \circ \tilde{f}^{\circ j}\right)(z)}
$$

in the intersection of the attracting and repelling regions for $\tilde{f}$ (note that (3.13) is simply the product form of Equation (ii) in Theorem 1.2). Define the mapping $H^{1}(z, w)=(h(z), w)$, and note that

$$
\left(\left(H^{1}\right)^{-1} \circ F \circ H^{1}\right)(z, w)=F_{1}(z)=(\tilde{f}(z), \lambda w(1+(g \circ h)(z))) .
$$

Again, the $(m-1)$-jet of $g \circ h$ is equal to $g^{*}$.

We define

$$
S=\bigcup_{j=1}^{m-1} S_{2 j-1}, \quad \widetilde{S}=\bigcup_{j=1}^{m-1} S_{2 j},
$$

where $S_{j}$ are the sectors defined in (1.4). Note that $S$ lies in the attracting region of $\tilde{f}$, while $\widetilde{S}$ lies in its repelling region. We first construct a mapping of the form $H_{1}^{2}(z, w)=\left(z, w k_{1}(z)\right)$, with $\lim _{|z| \rightarrow 0} k_{1}(z)=1$, which conjugates the mapping $F_{1}$ given in (3.14) with

$$
F_{\tilde{f}}(z, w)=\left(\tilde{f}(z), \lambda w\left(1+g^{*}(z)\right)\right)
$$

on the set $S \times U^{\prime}$, where $U^{\prime}$ is a small neighborhood of the origin in $\mathbf{C}^{n}$. We consider the equation $F_{1} \circ H_{1}^{2}=H_{1}^{2} \circ F_{\tilde{f}}$ (this is legitimate, since the mapping $H_{1}^{2}$ is clearly invertible), which in turn yields the functional equation

$$
\left(k_{1} \circ \tilde{f}\right)(z)=k_{1}(z) \frac{1+(g \circ h)(z)}{1+g^{*}(z)} .
$$

By iterating (3.17) by $\tilde{f}$ on the right and taking limits (again legitimate, since $S$ attracts $\tilde{f})$, we obtain

$$
k_{1}(z)=\prod_{j=0}^{\infty}\left(\frac{1+\left(g \circ h \circ \tilde{f}^{\circ j}\right)(z)}{1+\left(g^{*} \circ \tilde{f}^{\circ j}\right)(z)}\right)^{-1} .
$$

The uniform convergence of this product on compact subsets of $S$ follows from Result 2; we sketch the proof here. First, we note that the quotient on the righthand side of (3.18) can be written

$$
\frac{1+\left(g \circ h \circ \tilde{f}^{\circ j}\right)(z)}{1+\left(g^{*} \tilde{f}^{\circ j}\right)(z)}=1+\left(g_{1} \circ f^{\circ j}\right)(z),
$$


where $\left|g_{1}(z)\right| \leq C|z|^{m}$. We can write, for any $j=1,2, \ldots, f^{\circ j}(z)=\left(\tilde{h}^{-1} \circ f_{0, m}^{\circ j} \circ\right.$ $\tilde{h})(z)$, where $f_{0, m}$ will denote the time-one mapping of the vector field $V_{m}(z)=$ $z^{m} \frac{\partial}{\partial z}$. Moreover, fixing $0<\varepsilon \ll 1$, we choose $\tilde{h}$ so that it satisfies the estimates

$$
\tilde{h}(z)=z+O\left(z^{(m-1)+\varepsilon}\right), \quad \tilde{h}^{-1}(z)=z+O\left(z^{(m-1)+\varepsilon}\right) .
$$

Equation (3.20) allows us to write the right-hand side of (3.19) as

$$
1+\left(g_{1} \circ f^{\circ j}\right)(z)=1+\left(\hat{g}_{1} \circ f_{0, m}^{\circ j} \circ \tilde{h}\right)(z),
$$

where $\left|\hat{g}_{1}(z)\right| \leq C|z|^{(m-1)+\varepsilon^{\prime}}$ for some $C>0$ and $\varepsilon^{\prime}>0$. Combining these two facts, it is not hard to see that the product $k_{1}$ in (3.18) does indeed converge uniformly on compact subsets of $S$, and hence defines a holomorphic function $k$. Moreover, as $|z| \rightarrow 0$, we have $k_{1} \rightarrow 0$.

Now, working with the mapping $\widetilde{F}$, we can, in like manner, construct a mapping $\widetilde{H}_{1}^{2}(z, w)=\left(z, w \tilde{k}_{1}(z)\right)$ so that $\left(\widetilde{H}_{1}^{2}\right)^{-1} \circ \widetilde{F} \circ \widetilde{H}_{1}^{2}=F_{\tilde{f}}$. Note that

$$
\tilde{k}_{1}(z)=\prod_{j=0}^{\infty}\left(\frac{1+\left(\tilde{g} \circ \tilde{f}^{\circ j}\right)(z)}{1+\left(g^{*} \circ \tilde{f}^{\circ j}\right)(z)}\right)^{-1} .
$$

We can then define the mapping $H_{1}=H^{1} \circ H_{1}^{2} \circ\left(\widetilde{H}_{1}^{2}\right)^{-1}$ on the region $S \times U^{\prime}$ which conjugates $F$ to $\widetilde{F}$. Note that if we write $H_{1}(z, w)=(h(z), w k(z))$, then

$$
k(z)=\prod_{j=0}^{\infty}\left(\frac{1+\left(g \circ h \circ \tilde{f}^{\circ j}\right)(z)}{1+\left(\tilde{g} \circ \tilde{f}^{\circ j}\right)(z)}\right)^{-1} .
$$

On the other hand, in the region $\widetilde{S} \times U^{\prime}$, we can construct mappings $H_{2}^{2}(z, w)=$ $\left(h(z), w k_{2}(z)\right)\left(\operatorname{resp} . \widetilde{H}_{2}^{2}(z, w)=\left(z, w \tilde{k}_{2}(z)\right)\right)$ conjugating $F(\operatorname{resp} . \widetilde{F})$ with the mapping $F_{f}$, and thus obtain a mapping $H_{2}(z, w)=\left(H^{1} \circ H_{2}^{2} \circ\left(\widetilde{H}_{2}^{2}\right)^{-1}\right)(z, w)=$ $(h(z), w \hat{k}(z))$ which conjugates $F$ with $\widetilde{F}$ in this region. In this case, we have

$$
\hat{k}(z)=\prod_{j=1}^{\infty} \frac{1+\left(g \circ h \circ \tilde{f}^{\circ(-j)}\right)(z)}{1+\left(\tilde{g} \circ \tilde{f}^{\circ(-j)}\right)(z)} .
$$

Finally, in order to construct a holomorphic mapping $H$ defined in a full neighborhood of the origin in $\mathbf{C}^{2}$ conjugating $F$ with $\widetilde{F}$, we simply define

$$
H(z, w)= \begin{cases}H_{1}(z, w) & \text { if }(z, w) \in S \times U^{\prime} \\ H_{2}(z, w) & \text { if }(z, w) \in \widetilde{S} \times U^{\prime}\end{cases}
$$

Note that this mapping is well defined. For this to be true, we must have that $k$ agrees with $\hat{k}$ in the region $S \cap \widetilde{S}$. That is, we must have

$$
\prod_{j=0}^{\infty}\left(\frac{1+\left(g \circ h \circ \tilde{f}^{\circ j}\right)(z)}{1+\left(\tilde{g} \circ \tilde{f}^{\circ j}\right)(z)}\right)^{-1}=\prod_{k=1}^{\infty} \frac{1+\left(g \circ h \circ \tilde{f}^{\circ(-k)}(z)\right)}{1+\left(\tilde{g} \circ \tilde{f}^{\circ(-k)}\right)(z)} .
$$

But after unravelling this equation, we see that it is precisely the condition (3.13). Theorem 1.2 is proved. 


\section{REFERENCES}

[1] M. Abate, Discrete local holomorphic dynamics. Proceedings of 13th Seminar on Analysis and Its Applications, 1-31, Isfahan Univ. Press, Isfahan, 2003. MR2114495 (2005i:37049)

[2] P. Ahern and J.-P. Rosay, Entire functions, in the classification of differentiable germs tangent to the identity, in one or two variables, Trans. Amer. Math. Soc. 347 (1995), 543-572. MR1276933 (95d:30038)

[3] V. I. Arnold, Geometrical Methods in the Theory of Ordinary Differential Equations, Springer-Verlag, New York, 1983. MR695786 (84d:58023)

[4] C. Camacho, On the local structure of conformal mappings and holomorphic vector fields in C $^{2}$, Bull. Soc. Math. de France Astérisque 59-60 (1978). MR542732 (81d:58016)

[5] Y. S. Il'yashenko, Nonlinear Stokes Phenomena. Adv. in Soviet Math., vol. 14, Amer. Math. Soc., Providence, RI, 1993. MR1206041 (94d:32031)

[6] J. Milnor, Dynamics in One Complex Variable, Introductory Lectures, Vieweg, Braunschweig, 1999. MR1721240 (2002i:37057)

[7] A. A. Shcherbakov, Topological classification of germs of conformal mappings with identical linear part, Vestnik Moskov. Univ. Ser. I Mat. Mekh., 1982, no. 3, 52-57; English transl. in Moscow Univ. Math. Bull. 37 (1982). MR671059 (84f:58014)

[8] S. M. Voronin, Analytic classification of germs of conformal maps $(\mathbb{C}, 0) \rightarrow(\mathbb{C}, 0)$ with identical linear part. Funct. Anal. Appl. 15 (1981), 1-17. MR609790 (82h:58008)

Department of Mathematics, Purdue University, West Lafayette, Indiana 47906

E-mail address: majenkin@math.purdue.edu 